\title{
Effects of a Perturbation-Based Balance Training on Compensatory Postural Responses to Backward Loss of Balance in Patients with Cerebellar Disease: A Case Study
}

\author{
Chang-Ha Im¹, Jin-Hoon Park1,** \\ ${ }^{1}$ Department of Physical Education, Korea University, Korea
}

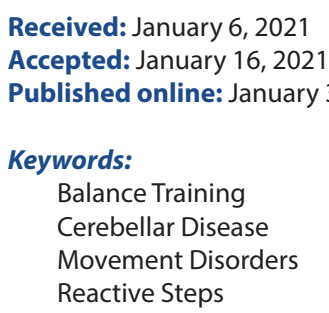

Received: January 6, 2021

Accepted: January 16, 2021

Keywords:

Balance Training

Cerebellar Disease

Movement Disorders

Reactive Steps

Published online: January 31, 2021
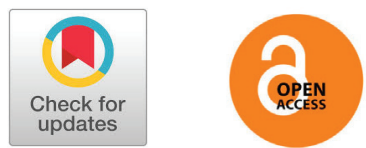

\section{ABSTRACT}

OBJECTIVES The damage to the cerebellum primarily results in balance-related abnormalities that may affect performance of locomotion and postural adjustments, eventually contributing to an increased risk of fall and fear of falling in patients with cerebellar disease. The purpose of the present study was to investigate the effect of a perturbation-based training that induced backward loss of balance on compensatory postural responses in patients with cerebellar disease.

METHODS The participant was a 51-year-old female diagnosed with spinocerebellar ataxia and had the disease for 19 years. The perturbation-based backward balance training was performed over 8 weeks (a total of 24 training sessions) in order to facilitate the perception of postural perturbation onset and execution of rapid compensatory responses.

RESULTS The patient demonstrated a noticeable reduction in the number of steps required to recover body balance after postural disturbances. The reduction of multi-step reactions in recovering balance could be attributed to improvements in the body center-of-mass displacement and trunk control during the landing of step. Besides, there were also improvements in subjective measures of functional mobility and psychological well-being after the balance training.

CONCLUSION Although current research evidence of balance rehabilitation for cerebellar patients is lacking, this study offers the feasibility of adaptive training to improve postural stability through task-specific training intervention.

(c) The Asian Society of Kinesiology and the Korean Academy of Kinesiology

\section{Introduction}

The cerebellum is a neural structure that plays a crucial role in regulating gait, postural stability, and coordination of movements. Particularly, the medial region of the cerebellum (i.e., vermis) is known to be greatly involved in maintenance of upright balance and stance [1]. Neurophysiological studies

*Correspondence: Jin-Hoon Park, Ph.D.; Department of Physical Education, Korea University; Anam-dong, Seongbuk-gu, Seoul 136-701, South Korea; Tel: 82-2-32902312; E-mail: jpark12@korea.ac.kr revealed that the medial cerebellar zone integrates spinal and vestibular inputs, and affects the neural pathways for balance control [2]. Accordingly, damage or lesions to this region primarily result in balance-related abnormalities that may influence performance of postural adjustments. These motor dysfunctions are known to significantly contribute to an increased risk of fall and fear of falling in patients with cerebellar disease [3]. In fact, it has been reported that cerebellar patients are more likely to experience more than 
one fall annually [4]. These falls frequently led to injuries or immobility in physical activity causing reduced quality of life in patients with cerebellar disease [5].

A fall is essentially a failure to regain balance from a postural instability [6]. Many studies have attempted to develop effective fall prevention programs, and it is suggested that exercise-based programs with balance training appear to be optimal rehabilitation interventions in preventing falls in older adults [7]. Particularly, task-specific balance training based on the objective analysis of key components in recovering balance could have more positive influence on fall prevention [8].

Although there are many factors that contribute to balance recovery from falls, it has been shown that execution of rapid and effective stepping responses upon postural disturbances is critical to prevent falls. Particularly, the characteristics of the first step movement after loss of balance, such as stepping time and length, are associated with successful recovery of body stability [9]. In addition, another key factor is the control of trunk movement following perturbation. Sufficient flexion of trunk motion in the opposite direction to the perturbation is also required to arrest the body center of mass (COM) for recovering stability [10]. Therefore, it is evident that exercise training programs that aimed to improving both capabilities of executing rapid stepping reactions and controlling trunk motion would produce greater reduction in fall rates.

It is reported that the most common causes of fall incidence in elderly population in daily life are associated with tripping or slipping [11]. In case of slipping that mostly induces a posterior fall, rapid compensatory responses are necessary to avoid a fall. Backward loss of balance is more dangerous than other directions of falls because the limit of foot stability is shorter than anterior direction and direct vision of environment is not available. Thus, adequate sensation of the posterior instability and subsequent execution of backward reactive stepping are important to prevent posterior falls [12].

Previous studies have demonstrated that repeated exposure to slipping conditions resulted in a reduction in step reaction time and an increase in step distance, and perturbationbased training that induces backward balance loss enhanced the capability to recover from posterior instability and subsequently reduced the likelihood of fall incidence in older adults [13]. The positive effects of training of the perturbation responses on adaptive control of postural balance were also observed in people with stoke and Parkinson's disease [14,15].

Although previous research has provided convincing evidence of the improved reactive responses upon backward postural disturbances, there is little research investigating such training effects in patients with cerebellar disease who suffer primarily from postural imbalance and coordination deficits. The reasons for the lack of study on the effectiveness of interventions for patients with cerebellar disease could be due to the facts that the number of cerebellar patient population is relatively small and the underlying causes of disease and symptoms are diverse as compared with other neurological diseases [16]. Although there is some debate about the effects of rehabilitation training for balance improvement in cerebellar dysfunction, recent studies showed that intensive balance training could enhance functional recovery of balance in cerebellar patients, and these changes were medicated by the neural plasticity in the remaining intact parts of the cerebellum and remote brain areas such as premotor cortex $[17,18]$. These promising results suggest that patients with cerebellar disease may preserve residual learning capacity and can demonstrate positive outcomes in balance function through training programs. The aim of the present study was to investigate the effect of a perturbation-based training that induced backward loss of balance on compensatory postural responses and functional mobility in patients with cerebellar disease.

\section{Methods}

\section{Participant}

The patient was a 51-year-old, right-handed female (height: $168 \mathrm{~cm}$, weight: $59 \mathrm{~kg}$ ) living with her husband and a daughter. She was diagnosed with spinocerebellar ataxia (SCA), a genetic and degenerative disease in which neurons in the cerebellum become progressively deteriorated over time. Her neurological condition was confirmed by CT/MRI scans and neurologist's diagnosis at the age of 32. She showed several motor symptoms such as a wide-based and unsteady gait 
and balance, uncoordinated limb movement, mild postural tremor, and slurred speech. She had no visual or orthopedic problems that would affect standing or walking at least $10 \mathrm{~m}$ without assistance. She received no physiotherapy intervention related to balance training in the past. The Mini Mental State Examination (MMSE) score was higher than 25, thus she was cognitively normal. The degree of motor symptoms was measured by the International Cooperative Ataxia Rating Scale (ICARS) and the Scale for the assessment and rating of ataxia (SARA). She was independent in most of daily activities, but particularly had difficulties in turning or stepping over obstacles. She was taking no medications during the study period. Informed consent for participation was obtained from the patient according to the Declaration of Helsinki. This study was approved by the Human Research Ethics Committee of Korea University (KUIRB-2019-0141-01).

\section{Experimental design and procedure}

This case study used an A-B-A type design with a single subject of cerebellar patient. The outcome measures were conducted three times; pre-test $(\mathrm{A})$ one week before the intervention, post-test (B) one week after the end of the intervention, and follow-up test (A) 4 weeks after the completion of the intervention. We assessed clinical and kinematic outcomes in each testing period. We measured the compensatory recovery responses with using a backwarddirected lean-and-release test. A horizontal cable was connected to the participant and she kept her body inclined in a backward direction. Then, the cable was released after a random time delay, and she was instructed to recover balance from a posterior fall in a natural way. The perturbation magnitude of backward lean was selected between 1 and 5\% of her body weight. The magnitude was monitored using a load cell connected to the cable. She wore a suspended safety harness attached to the ceiling to protect from a fall to the floor. External assistance by a researcher was provided if she was unable to recover balance alone. No practice trials were allowed, and 4 trials for each magnitude were presented randomly to minimize anticipatory actions. In order to measure compensatory stepping responses and trunk motion, we analyzed the outcomes only from the two largest lean magnitudes (i.e., 4 and 5\% BW), from which the participant took at least one step on average to recover balance from the perturbation.

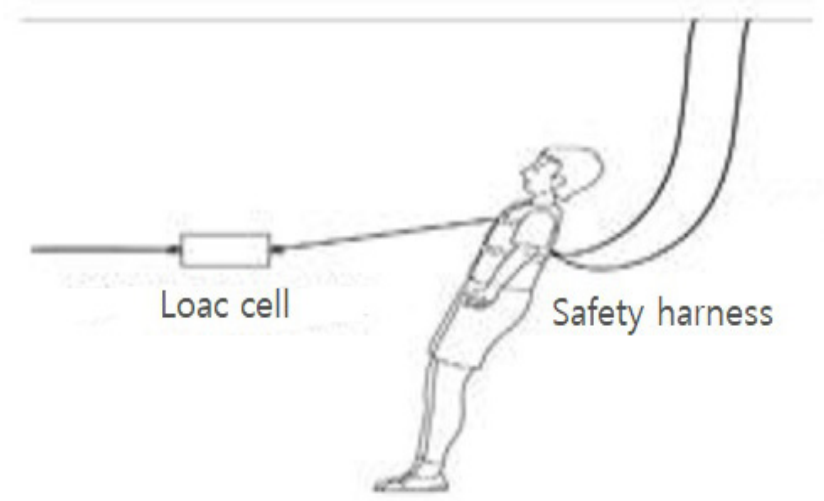

Figure 1. Schematic diagram of experimental setup for posterior fall trials in the lean and release method.

\section{Intervention}

The perturbation-based backward balance training sessions were lasted 30 minutes, 3 times per week for 8 weeks (a total of 24 training sessions). The duration and frequency of the current intervention were adopted from previous studies showing improved balance functions with similar training schedule [19]. The aim of intervention was to regain body balance by facilitating the perception of postural perturbation onset and execution of rapid compensatory responses, since it was previously found that rapid onset detection through integration of sensory information and subsequent motor response are critical to avoid falls from unexpected perturbations [20]. Specifically, the postural balance training focused on enhancing conscious awareness of body motion and simultaneous development of rapid and long reactive steps and compensatory trunk movements to arrest an ongoing fall in the posterior direction.

Initially, the intervention began with a slow backward walking while holding both hands of an instructor facing each other. The patient was encouraged to relax and walk backward in a stable and controlled manner and the instructor's support helped guiding the speed and direction of stepping movements. This volitional backward stepping session allowed the patient to become familiar with the required task and posture [21]. Following approximately 10minutes of backward 
walking practice, the remaining 20 minutes of the training were assigned to the perturbation-based balance training provided manually by an instructor. The patient was first asked to stand with her feet about shoulder-width apart, and a heavy-duty resistance band was placed around her back under her arms at the chest level. The instructor stood in front of her while holding held both ends of the band and adjusted the appropriate distance to maintain a suitable band tension. The patient was then asked to lean backward slightly and to keep her body straight. Perturbations were provided by releasing the band, which induced a posterior fall from a static inclined position, and the patient was instructed to react naturally but to take steps and flex trunk rapid and long enough to stop the backward motion of body. This training session under external perturbation aimed at improving feedback postural reaction [22]. During the course of the training sessions, perturbation magnitude was progressively increased by adjusting the lean angle in order to maximize the effect of training [23]. The instructor had considerable knowledge and experience in movement disorders due to cerebellar dysfunctions and supervised all sessions with the help of one trained assistant for safety.

\section{Outcome measures}

Balance recovery performance data were collected using an OPTITRACK 3D camera system (NaturalPoint Inc., Oregon, USA) at sampling frequency with $120 \mathrm{~Hz}$. A 14-body segment (feet, shanks, thighs, trunk, head and neck, upper arms, lower arms and hands) 3D model was constructed by reflective markers placed on selected body landmarks. The total body center of mass (COM) was calculated as the weighted sum of the COM of each body segment based on the anthropometric data compiled by Winter [24]. Kinematic characteristics of stepping movements of lower limbs and whole-body COM variables were analyzed using MATLAB (Mathworks, Natick, MA). Step initiation was calculated with reference to the vertical velocity profile of the foot position data. Foot lift was defined as the moment when the velocity exceeded 5\% of peak velocity and stayed over this threshold for at least 50 $\mathrm{ms}$. The moment at which the velocity became less than $5 \%$ of maximum after the peak velocity point was determined as foot landing. Based on these landmarks, the following kinematic variables of the initial step were collected: step reaction time (the interval from release of the cable until the initiation of a step), step response time (the interval between liftoff and landing of the foot), step distance (the sagittal distance between the initiation and landing of a step), and number of steps until restoring balance.

Furthermore, the following COM-related variables were also analyzed upon the landing of the initial step: foot-COM distance (the sagittal plane perpendicular distance between the landed foot and the vertical projection of $\mathrm{COM}$ ), $\mathrm{COM}$ velocity (the value of the first derivative of the anterioposterior COM displacement at foot landing), and trunk angular displacement (sagittal change of trunk angle relative to the vertical direction from the release of cable until the landing of initial foot).

In addition, we evaluated subjective measures of balance using the Berg Balance Scale (BBS) and the Fall Efficacy Scale (FES). The BBS assesses the balance performance in 14 functional tasks related to daily activity [25] and the FES measures fear of falling in performing 10 activities of daily living [26]. Besides, psychological outcomes were measured using the Beck Depression Index (BDI) and Quality of life (QOL). The BDI consists of 21 items evaluating the level of clinical depression [27] and the QOL measures physical and mental health, and social and environmental relationships [28].

\section{Results}

The patient attended all 24 training sessions over an 8 -week period of the postural balance program. Her overall motor symptoms evaluated by the clinical scale ICARS (36 points with a maximum score of 100) and SARA (17 points with a maximum score of 40) almost did not change over the training period.

The outcomes of stepping performance over each testing period were shown in $<$ Figure $2>$. The number of steps to recover balance was 4.38 before training. It was required almost 4 backward steps for her to regain the body balance completely from the postural perturbation. After 8 weeks of 

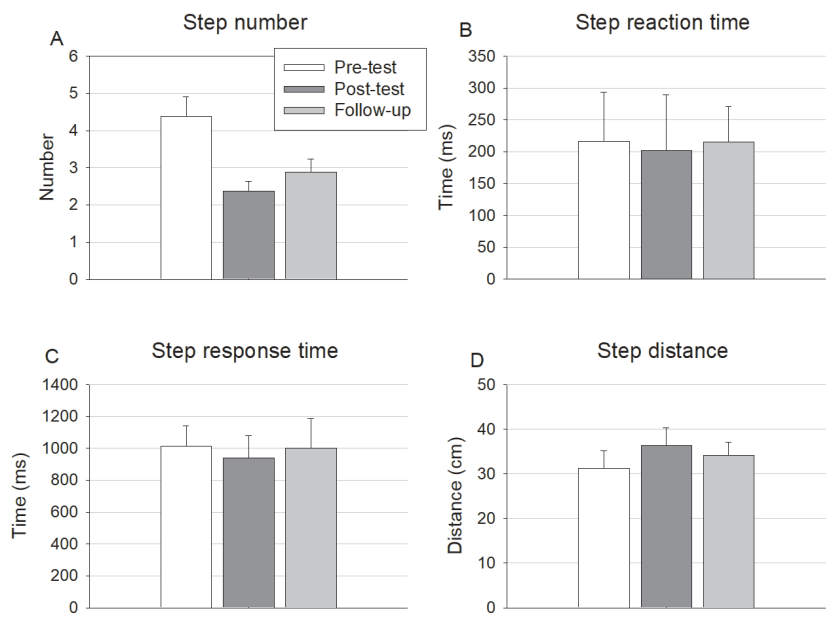

Figure 2. Plots of step number (A), step reaction time (B), step response time (C), and step distance (D) across the testing period.

training, she improved the number of steps to 2.34. She was able to stop body motion and restore balance with almost 2 steps for the same perturbation magnitude. The number of steps was 2.88 at follow-up test. She demonstrated a slight decline in step number 1 month after the end of training $<$ Figure $2 \mathrm{~A}>$. Step reaction time, which is the interval from cable release to the initiation of a foot lift, was $216 \mathrm{~ms}$ before training. This reaction time was slightly decreased to $202 \mathrm{~ms}$ in the post-training test. However, it was then increased to 215 $\mathrm{ms}$ in the follow-up testing $<$ Figure $2 \mathrm{~B}>$. Step response time, the interval between liftoff and landing of the initial foot, was $1016 \mathrm{~ms}$ prior to training. The step response time was then decreased to $939 \mathrm{~ms}$ when measured in the post-intervention period. However, it was increased to $1003 \mathrm{~ms}$ in the follow-up testing $<$ Figure $2 \mathrm{C}>$. Step distance, the distance between the initiation and landing of the first step in the sagittal plane, was $31.25 \mathrm{~cm}$ in the pre-testing period. This distance was increased to $36.33 \mathrm{~cm}$ in the post-testing period. She was able to step longer after the postural balance training. Step distance was reduced to 34.09 in the follow-up test $<$ Figure $2 \mathrm{D}>$.

The kinematic measures of body COM and trunk motion during each testing period were presented in $\langle$ Figure 3$\rangle$. Foot-COM distance, the distance the sagittal distance between the stepping foot and body COM at the time of initial step landing, was $11.33 \mathrm{~cm}$ before training. The distance was increased to $19.54 \mathrm{~cm}$ after the 8-week postural training, and
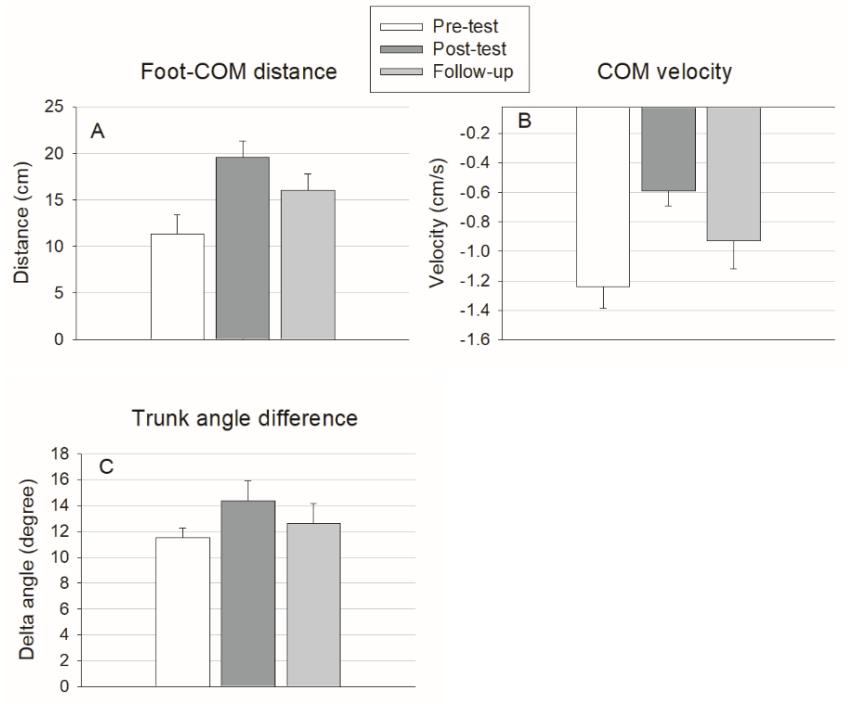

Figure 3. Plots of foot-COM distance (A), COM velocity (B), and trunk angle difference $(\mathrm{C})$ across the testing period.

then decreased to $16.04 \mathrm{~cm}$ in the follow-up test $<$ Figure $3 \mathrm{~A}\rangle$. $\mathrm{COM}$ velocity at the initial step landing was $1.24 \mathrm{~cm} / \mathrm{s}$ in the posterior direction before training. In the post-training test, the COM velocity was reduced to $0.59 \mathrm{~cm} / \mathrm{s}$, suggesting the movement of whole body became slower upon the landing of initial step. after the postural balance training. It was then increased to $0.93 \mathrm{~cm} / \mathrm{s}$ in the follow-up test $<$ Figure $3 \mathrm{~B}>$. Trunk angle difference, the change of trunk angle from the cable release until the landing of initial step in the sagittal plane, was 11.52 degree before training. Over the 8-week training period, the angle difference was increased to 14.38 degree, indicating the trunk was flexed more in the opposite direction to the perturbation at the landing of initial step. It was then decreased to 12.64 degree in the follow-up test $<$ Figure $3 \mathrm{C}>$.

The outcomes related to subjective measures of balance and psychological function are shown in $<$ Table $1>$. She demonstrated an improvement in the BBS by 6 points (17.1\%) over the 8-week training period, and this gain in the BBS score almost retained until the 1-month follow-up period. The FES score was increased by 12 points $(25.5 \%)$ from the pre- to post-training test. Her FES score was then decreased by 4 points from post-training to follow-up test. There was a reduction in the BDI by 8 points (34.8\%) from the pre- to post-training test. The BDI score was further decreased by 3 
Table 1. Training outcomes for self-reported functional mobility and psychological well-being.

\begin{tabular}{lcccc}
\hline Outcome measures & $\begin{array}{c}\text { Pre- } \\
\text { test }\end{array}$ & $\begin{array}{c}\text { Post- } \\
\text { test }\end{array}$ & $\begin{array}{c}\text { Follow- } \\
\text { up }\end{array}$ & $\begin{array}{c}\text { Change (\%) } \\
\text { Pre- post- } \\
\text { test }\end{array}$ \\
\hline Berg Balance Scale & $35 / 56$ & $41 / 56$ & $40 / 56$ & 17.1 \\
Fall Efficacy Scale & $47 / 100$ & $59 / 100$ & $55 / 100$ & 25.5 \\
Beck Depression Index & $23 / 63$ & $15 / 63$ & $12 / 63$ & -34.8 \\
Quality of Life & $89 / 130$ & $102 / 130$ & $107 / 130$ & 14.6 \\
\hline
\end{tabular}

more points from the post-training to follow-up test period. She also showed an improvement in the QOL by 13 points (14.6\%) from the pre- to post-training test, and continued improvement by 5 more points in the QOL score 1 month after the completion of training.

\section{Discussion}

This case study investigated how an 8-week period of postural balance training influences control of compensatory responses in a patient with cerebellar disease. We particularly focused on the effects of task-specific balance training on the posterior reactive stepping and trunk movements to recover balance from postural perturbations. The results indicated that there were improvements in nearly all outcomes following the 8 -week postural balance training. The patient demonstrated a noticeable reduction ( $46.5 \%$ change) in the number of steps required to recover body balance after postural disturbances. The stepping response results showed decreases in step reaction time (6.4\% change) and step response time $(7.5 \%$ change), and an increase in step distance (16.2\% change). In addition, the data associated with the whole body movement showed increases in foot-to-COM distance (72.4\% change) and trunk angle difference (24.8\% change), and a reduction in COM velocity (52.4\% change). Besides, there were also improvements in subjective measures of functional mobility and psychological well-being after the balance training. Whereas most of the positive training effects were diminished over the 1-month follow-up period, gains in psychological benefits were sustained 1 month after the completion of the training.
Our results showed that the balance training employed in this study led to a substantial improvement in reducing the number of steps required to restore balance from perturbation. It is known that rapid perception of the perturbation onset and execution of appropriate stepping actions are crucial to avoid falls when responding to the postural instability [14]. Although there were improvements in spatiotemporal characteristics of step execution following the balance intervention, we found less gains in stepping-related outcomes as compared to those of trunk-related movements. These small changes may result from the nature of movement task adopted and executed by the patient in the current study. Previous studies reported that reactive stepping movement in response to a sudden external perturbation is mostly governed by lower lever of neural control (e.g., brainstem or spinal cord) [29]. Thus, upper neural centers including cerebellum are not significantly involved in producing reactive stepping movements. In support of this report, it has been shown that cerebellar patients virtually preserve the capability to execute rapid compensatory stepping reactions when responding to postural perturbations [30]. Therefore, the reason for the relatively small training benefit in stepping responses may reside in such neural mechanisms underlying the control of reactive stepping movements.

In addition to stepping response, the control of body COM and trunk motion is also considered as a key component for effective regaining of postural stability from destabilizing disturbance [10]. We found relatively large improvements in COM-related variables after the balance training. It is thus reasonable that the reduction of multi-step reactions in recovering balance observed in this study could be attributed to improvements in COM displacement and trunk control during the landing of step. The enhanced capabilities to control body COM and trunk motion by the balance training possibly contribute to more effective attenuation of body momentum, thus facilitating postural control from balance perturbations.

The observed finding could be explained by the fact that cerebellar diseases commonly affect coordination of multijoint movements such as limb and trunk movements [31], which may the cause for the poor control of torso motion and consequent failure of successful recovery of balance. However, 
the task-specific intervention targeting such coordination and balance impairments due to cerebellar disease could lead to promotion of the patient's capacity to control trunk motion and subsequently reduce the number of steps to recover balance. This description is consistent with previous studies reporting that therapeutic training strategies relevant to the specific functional goals or outcomes can have more beneficial effects on motor recovery than nonspecific rehabilitation training [32].

The potential mechanisms underlying functional improvement after the training have not been extensively documented in patients with cerebellar disease. Recent studies revealed that perturbation-based training induces an improved intersegmental coordination strategy that may promote dynamic stability and specifically the ability of controlling foot movement during balance recovery responses in order to compensate for the impaired cerebellar functions [33,34]. In addition, studies on neural rehabilitation have suggested that although cerebellar damages can induce poor recovery or low benefit of rehabilitation training, it has been shown that cerebellar patients still able to adapt or relearn motor coordination [17]. Burciu and colleagues [18] exhibited the neural changes associated with improvements of motor function after two weeks of sensorimotor balance training in patients with cerebellar disease. They found that the learning impairments because of cerebellar dysfunction are compensated by other part of the brain and also by remaining cerebellar areas less affected by the disease. Moreover, a recent study using fMRI demonstrated that not only motor and sensory brain areas, but prefrontal area was also greatly engaged in improved reactive balance control, which indicates that higher-level processing such as planning and modulating reactive responses is associated with adaptation to external perturbations [35]. Taken together, it can be speculated that these proposed compensatory neural mechanisms might be attributed to the observed gains in reactive balance functions after perturbation training in the cerebellar patient.

Our findings also demonstrated that the 8-week balance intervention also had a positive effect on the patients' depression and QOL level. In general, cerebellar patients are frequently accompanied by depressive symptoms. Nonetheless, the use of motor rehabilitation approaches such as the postural balance training in the current study diminished the symptoms of depression and perceived qualify of life. It is possible that these improvements may be due to increased confidence toward balance and mobility in everyday activities, and social and supportive relationships with experimenters during her visit for the training. The training effects on psychological well-being were even evident 1 month later at the follow-up test. However, it should be noted that the motor and functional improvements were not sustained over the follow-up period, which emphasizes the importance of continuous rehabilitative training for long-term maintenance of therapeutic benefits in patients with cerebellar disease.

\section{Conclusions}

The present study demonstrated that the perturbationbased balance training may be helpful for improving compensatory postural responses following backward loss of balance in patients with cerebellar disease. Although current research evidence of balance rehabilitation for cerebellar patients is lacking, this study offers the feasibility of adaptive training to improve postural stability through task-specific training intervention. The major limitation of the study is that general inferences cannot be drawn from a report of one patient's case. Thus, future research should attempt to investigate the effectiveness of this rehabilitation training with a larger sample of patients to provide further confirmation. In addition, future work also needs to examine neural substrates the for insight into pathophysiological mechanisms underlying the beneficial effects of perturbation-based balance training.

\section{Acknowledgments}

This research was supported by the College of Education, Korea University Grant in 2020.

\section{Conflicts of Interest}

The authors declare no conflict of interest. 


\section{References}

1. Morton SM, Bastian AJ. Cerebellar control of balance and locomotion. Neuroscientist. 2004; 10(3):247-259.

2. Asanuma C, Thach WT, Jones EG. Distribution of cerebellar terminations and their relation to other afferent terminations in the ventral lateral thalamic region of the monkey. Brain Res Rev. 1983; 5:237-265.

3. van de Warrenburg BP, Steijns JA, Munneke M, Kremer BP, Bloem BP. Falls in degenerative cerebellar ataxias. Mov Disord. 2005; 20:497-500.

4. Fonteyn EM, Schmitz-Hübsch T, Verstappen CC, et al. Falls in spinocerebellar ataxias: Results of the EuroSCA Fall Study. Cerebellum. 2010; 9:232-239.

5. Schmitz-Hübsch T, Coudert M, Giunti P, et al. Self-rated health status in spinocerebellar ataxia results from a European multicenter study. Mov Disord. 2010; 25:587595.

6. Horak FB. Postural orientation and equilibrium: what do we need to know about neural control of balance to prevent falls? Age Ageing. 2006; 35(2):7-11.

7. Grabiner MD, Donovan S, Bareither ML, et al. Trunk kinematics and fall risk of older adults: translating biomechanical results to the clinic. J Electromyogr Kinesiol. 2008; 18:197-204.

8. Bayona NA, Bitensky J, Salter K, et al. The role of taskspecific training in rehabilitation therapies. Top Stroke Rehabil. 2005; 12:58-65.

9. Maki BE, McIlroy WE. The control of foot placement during compensatory stepping reactions: does speed of response take precedence over stability? IEEE Trans Rehabil Eng. 1999; 7:80-90.

10. Pavol MJ, Owings TM, Foley KT, Grabiner MD. Mechanisms leading to a fall from an induced trip in healthy older adults. J Gerontol A Biol Sci Med Sci. 2001; 56:M428-437.

11. Cumming RG, Klineberg RJ. Fall frequency and characteristics and the risk of hip fractures.

12. Luchies CW, Alexander NB, Schultz AB, Ashton-Miller J. Stepping responses of young and old adults to postural disturbances: kinematics. J Am Geriatr Soc.1994;
42:506-512.

13. Gerards MH, McCrum C, Mansfield A, Meijer K. Perturbation-based balance training for falls reduction among older adults: Current evidence and implications for clinical practice. Geriatr Gerontol Int. 2017; 17(12):2294-2303.

14. Chen Z, Ye X, Chen W, et al. Effectiveness of backward walking for people affected by stroke. Medicine. 2020; 99(27):1-8.

15. Jobges M, Heuschkel G, Pretzel C, et al. Repetitive training of compensatory steps: a therapeutic approach for postural instability in Parkinson's disease. J Neurol Neurosurg Psychiatry. 2004; 75:1682-1687.

16. Marsden J, Harris C. Cerebellar ataxia: pathophysiology and rehabilitation. Clin Rehabil. 2011; 25:195-216.

17. Ilg W, Synofzik M, Brotz D, et al. Intensive coordinative training improves motor performance in degenerative cerebellar disease. Neurology. 2009; 73:1823-1830.

18. Burciu RG, Fritsche N, Granert O, et al. Brain changes associated with postural training in patients with cerebellar degeneration: a voxel-based morphometry study. J Neurosci. 2013; 33:4594-4604.

19. Mansfield A, Peters AL, Liu BA, Maki BE. Effect of a perturbation-based balance training program on compensatory stepping and grasping reactions in older adults: a randomized controlled trial. Phys Ther. 2010; 90: 476-491.

20. Maki BE, McIlroy WE. Postural control in the older adult. Clin Geriatr Med. 1996; 12:635-658.

21. Grobbelaar R, Venter R, Welman KE. Backward compared to forward over ground gait retraining have additional benefits for gait in individuals with mild to moderate Parkinson's disease: a randomized controlled trial. Gait Posture. 2017; 58:294-299.

22. Smania N, Corato E, Tinazzi M, et al. Effect of balance training on postural instability in patients with idiopathic Parkinson's disease. Neurorehabil Neural Repair. 2010; 24:826-834.

23. Vickers JN, Livingston LF, Umeris-Bohnert S, Holden D. Decision training: the effects of complex instruction, variable practice and reduced delayed feedback on the 
acquisition and transfer of a motor skill. J Sports Sci. 1999; 17:357-367.

24. Winter DA. Biomechanics and motor control of human movement, 2nd ed. New York. Wiley-Interscience. 1990, p 51-74.

25. Berg K, Wood-Dauphinee S, Willizma JI. The balance scale: Reliability assessment with elderly residents and patients with an acute stroke. Scand J Rehab Med. 1995; 27:27-36.

26. Tinetti ME, Richman D, Powell L. Falls efficacy as a measure of fear of falling. J Gerontol. 1990; 45:239-243.

27. Beck AT, Ward C, Mendelson M, Mock J, Erbaugh J. An inventory for measuring depression. Arch General Psych. 1961; 4:561-571.

28. Group WHOQOL. Development of the World Health Organization WHOQOL-BREF quality of life assessment. Psych Med. 1998; 28:551-558.

29. Maki BE, Mcilroy WE. Control of compensatory stepping reactions: age-related impairment and the potential for remedial intervention. Physiother Theory Pract. 1999; 15: 69-90.
30. Timmann D, Horak FB. Perturbed step initiation in cerebellar subjects: 2 . Modification of anticipatory postural adjustments. Exp Brain Res. 2001; 141:110-120.

31. Bastian AJ, Martin TA, Keating JG, Thach WT. Cerebellar ataxia: abnormal control of interaction torques across multiple joints. J Neurophysiol. 1996; 76:492-509.

32. Hubbard IJ, Parsons MW, Neilson C, et al. Task-specific training: evidence for and translation to clinical practice. Occup Ther Int. 2009; 16:175-189.

33. Aprigliano F, Martelli D, Kang J, et al. Effects of repeated waist-pull perturbations on gait stability in subjects with cerebellar ataxia. J Neuroeng Rehabil. 2019; 16(1):50-61

34. Monaco V, Aprigliano F, Lofrumento M, et al. Uncontrolled manifold analysis of the effects of a perturbation-based training on the organization of leg joint variance in cerebellar ataxia. Exp Brain Res. 2020; doi:10.1007/ s00221-020-05965-x.

35. Kinomoto K, Takayama Y, Watanabe T, et al. The mechanisms of recovery from cerebellar infarction: an fMRI study. Neuroreport. 2003; 14:1671-1675. 